\title{
Motor and sensory conduction in different segments of the radial nerve in normal subjects
}

\author{
W. TROJABORG AND E. H. SINDRUP \\ From the Laboratory of Clinical Neurophysiology, University Hospital, Copenhagen
}

The rate of conduction along proximal motor fibres of the radial nerve was measured by Gassel and Diamantopoulos (1964) and their findings in normal subjects were confirmed by Tönnis (1965). Jebsen (1966b) found a $10 \%$ faster conduction in proximal than in distal fibres of the radial nerve with the respønse of the extensor indicis muscle as indicator. Downie and Scott (1964) have recorded sensory action potentials along the radial nerve with surface electrodes. Buchthal and Rosenfalck (1966) described spread of the potential to the radial from the median nerve at wrist to give apparently normal conduction in spite of lesions of the radial nerve.

The study presented in this report deals with conduction velocity along the radial nerve in normal subjects: in proximal and distal segments of motor fibres, and in proximal, intermediate and distal segments of sensory fibres. The spread of potentials from the median nerve to the different recording sites near the radial nerve was ascertained.

\section{MATERIAL AND METHOD}

Thirty-five subjects, 21 females and 14 males, 16 to 28 years old, without history, signs, or symptoms of neuromuscular involvement were examined.

MOTOR CONDUCTION The radial nerve was stimulated at three sites: (1) in the forearm, $8 \mathrm{~cm}$ proximal to the styloid process of the ulnar bone where the nerve can be palpated just lateral to the extensor carpi ulnaris muscle (Jebsen, 1966a); (2) at the elbow in the groove between brachioradialis and the biceps tendon, $6 \mathrm{~cm}$ proximal to the lateral epicondyle of the humerus; and (3) at the axilla in the groove between coracobrachialis and the medial edge of the brachial triceps, $18 \mathrm{~cm}$ proximal to the medial epicondyle of the humerus.

The stimulus was applied through needle electrodes insulated except for a $3 \mathrm{~mm}$ bared tip. The cathode was adjusted close to the nerve as indicated by the lowest threshold of the muscle action potential (0.4 mA, S.D. $0.2, \mathrm{~N}=22,0.2 \mathrm{msec}$ in duration). The supramaximal stimulus was at least five times the threshold current ( 2 to $4 \mathrm{~mA}$ ).

The action potentials were recorded with concentric needle electrodes (DISA 13K0511) adjusted to the region of maximal response in the brachial triceps, the brachioradial, the extensor digitorum communis, the extensor pollicis longus, and the extensor indicis muscles. The motor responses and the stimulus current were recorded on two channels of an electromyograph (DISA). Single traces with a time base of 0.5 or $1 \mathrm{msec} / \mathrm{mm}$ were used for measurement. The conduction time was measured from the stimulus to the onset of the action potential; the amplitude was measured peak-to-peak. The distance between the cathode at axilla and at elbow was measured with obstetric calipers (Gassel and Diamantopoulos, 1964).

SENSORY CONDUCTION The sensory fibres of the radiati nerve were stimulated (1) with surface electrodes, the? cathode placed at the proximal phalanx of the thumb anda the anode $20 \mathrm{~mm}$ distal to it; (2) with needle electrodes the cathode placed over the nerve at the wrist along the lateral border of the radial bone and the anode on the dorsum of the forearm at a transverse distance of 3 to 4 $\mathrm{cm}$ from the cathode. For comparison the nerve was stimulated at the same site with a bipolar surface electrode (DISA 13K62). The supramaximal stimulus was at least five times threshold, $48 \pm 2.5 \mathrm{~mA}(\mathrm{~N}=23)$ when stimulating the thumb, $17 \pm 1.9 \mathrm{~mA}(\mathrm{~N}=19)$ when stimulating at the wrist with surface electrodes, and $6 \pm 1.5 \mathrm{~mA}(\mathrm{~N}=13)$ when stimulating at the wrist with needle electrodes.

The sensory action potentials were recorded with needle electrodes as described by Buchthal and Rosenfalck (1966). At the axilla and elbow the electrodes positioned by means of the motor threshold were used for recording. At the wrist the 'near-nerve' electrode was placed with the amplitude of the sensory potential as indicator: (1) at the elbow evoked by stimulating the radial nerve at wrist; (2) at the wrist evoked by stimulating the thumb; and (3) at the wrist evoked by stimulating the mixed nerve at the elbow. The remote electrode was placed transversely to the 'near-nerve' electrode as far as possible from the median nerve. A second set of electrodes was used to record simultaneously from the median nerve with the same inter-electrode distance, the remote electrode being ulnar to the 'near-nerve' electrode.

The relation between motor threshold and amplitude of the sensory potential evoked by supramaximal stimuli showed that amplitudes did not vary systematically with the threshold as long as the threshold was below $1 \mathrm{~mA}$ and above $0.3 \mathrm{~mA}$ (Fig. 1). The highest amplitude was 


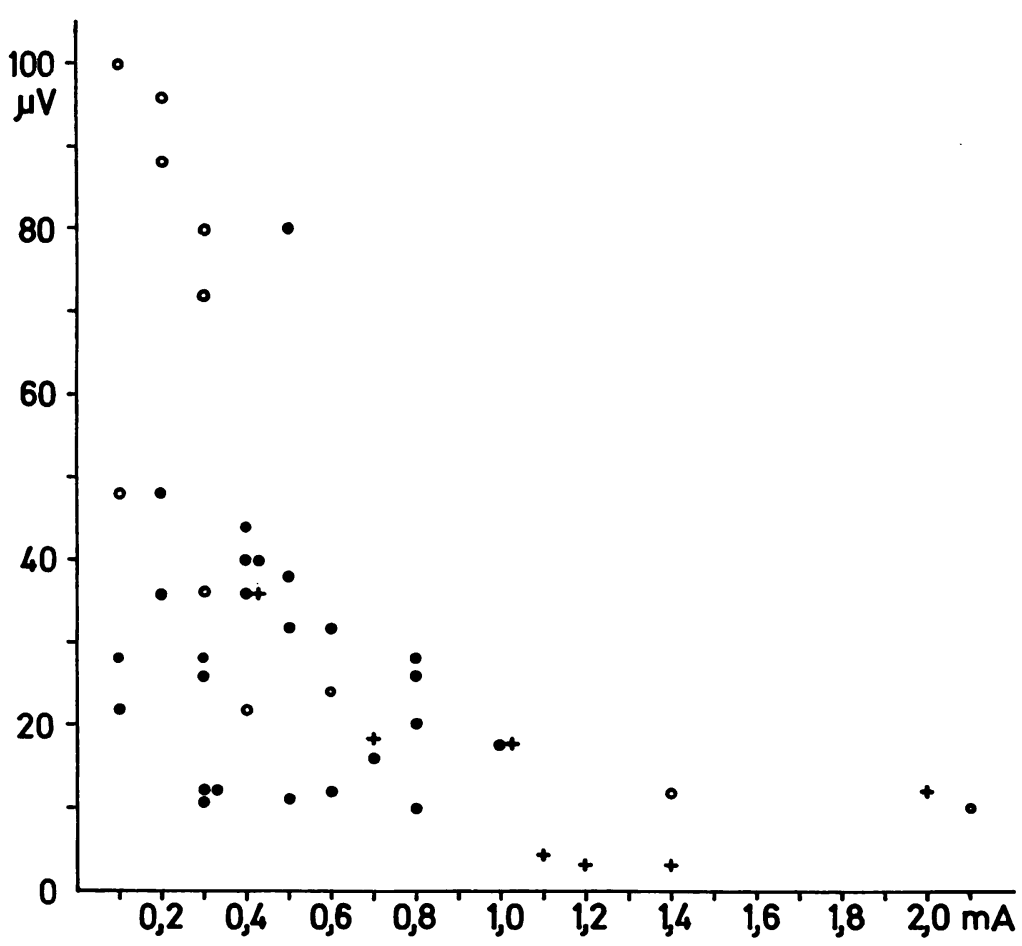

FIG. 1. Amplitude of the sensory action potentials evoked by supramaximal stimuli to the radial nerve at wrist $(11 \pm 2.9 \mathrm{~mA})$ as a function of the motor threshold of the extensor digitorum communis muscle stimulated at elbow. $=25$ subjects; $\bigcirc$ and + are two subjects in whom the electrode was approached stepwise to the radial nerve (stimulus $6 \mathrm{~mA}$ ). Temperature near the nerve $35^{\circ} \mathrm{C}$.

found when an antidromically conducted sensory potential occurred before the motor response. Single traces and 12 to 15 photographically superimposed traces were used for measurement. The time base was 0.25 or $0.5 \mathrm{msec} / \mathrm{mm}$.

The spread from the median to the radial nerve at elbow and axilla was studied in three subjects by electronic averaging of 500 to 1,000 responses together with a calibration signal (Andersen, 1966). For control the same number of sweeps were then averaged with the electrodes in position but without stimulus.

The conduction time was measured as the time interval between the onset of the stimulus and the initial positive peak of the sensory potential. The amplitude was measured peak-to-peak.

MIXED NERVE The needle electrodes placed near the nerve at the three sites along the extremity were also used for stimulating and recording from the mixed nerve.

To maintain an even temperature the hand, forearm, and arm were irradiated by an infra-red heater. The temperature was measured by a thermocouple on the surface of the skin near the nerve. The temperature averaged $32^{\circ} \mathrm{C}$ at the thumb (S.D. $1 \cdot 6^{\circ} \mathrm{C}$ ), $34^{\circ} \mathrm{C}$ at wrist (S.D. $1.5^{\circ} \mathrm{C}$ ), $35^{\circ} \mathrm{C}$ at the elbow (S.D. $0 \cdot 8^{\circ} \mathrm{C}$ ), and $36^{\circ} \mathrm{C}$ at axilla (S.D. $0 \cdot 4^{\circ} \mathrm{C}$ ).

\section{RESULTS}

MOTOR CONDUCTION (Fig. 2, Table I) The conduction velocity between axilla and elbow was $69 \mathrm{~m} / \mathrm{sec}$ whether measured in fibres to the brachioradial, extensor digitorum communis, extensor pollicis longus, or extensor indicis muscles. The conduction velocity between elbow and forearm was $10 \%$ lower than in the proximal segment of the nerve $(P<0.02)$. The same difference was found in the 'mixed' nerve: $70 \pm 1.4 \mathrm{~m} / \mathrm{sec}$ between elbow and axilla as compared with $61 \pm 1 \cdot 1 \mathrm{~m} / \mathrm{sec}(\mathrm{N}=10)$ between elbow and forearm.

SENSORY CONDUCTION (Fig. 3, Table II) Sensory and motor fibres conducted at the same rate between elbow and axilla. In the forearm the sensory velocity was slightly higher than the motor $(9 \%, P<0.05)$. Whether stimulating the nerve at the thumb with surface electrodes or at the wrist with needle or with surface electrodes the velocity between wrist-elbow and elbow-axilla was the same, about $17 \%$ higher than the distal velocity, a similar difference as in the median and ulnar nerves (Buchthal and Rosenfalck, 1966). The distal 'conduction velocity' in the radial nerve averaged $58 \pm 1.3 \mathrm{~m} / \mathrm{sec}$, similar to that in the median nerve of the same subjects (57 \pm 1.5 $\mathrm{m} / \mathrm{sec}$ ).

The amplitude of the sensory action potential near the radial nerve at the wrist averaged $13 \pm 1.6 \mu \mathrm{V}$ as compared with $44 \pm 3.5 \mu \mathrm{V}$ over the median 
nerve $(\mathrm{N}=23)$. The amplitude of sensory action potentials along the arm depended on the site of stimulation, being six times higher at the elbow and 2.5 times higher at the axilla when the stimuli were applied at the wrist than when applied to the thumb. The amplitudes were the same whether evoked by

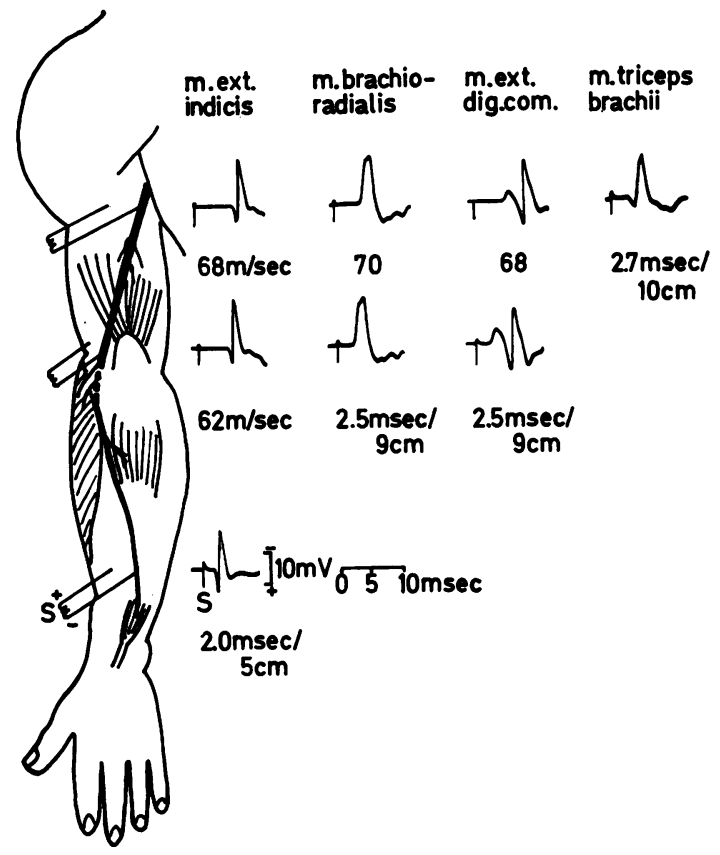

FIG. 2. Motor conduction velocity $(v, \mathrm{~m} / \mathrm{sec})$ and latency ( $m s e c, c m$ ) in the radial nerve. Action potentials from different muscles evoked by stimulating at axilla, elbow, and forearm. Subject: J.S.C., male, 16 years old. Temperature at the three sites near the nerve $35^{\circ} \mathrm{C}$. Stimulus $(S): 4 m A(5-8 \times t s)$. Distance between axilla and elbow $16 \mathrm{~cm}$, between elbow and forearm $18 \mathrm{~cm}$. surface or by needle stimulation at the wrist. When stimulating the thumb the amplitude decreased $60 \%$ from wrist to elbow and only a further $10 \%$ from elbow to axilla; when stimulating the wrist the amplitude decreased $70 \%$ from elbow to axilla.

THE ORIGIN OF THE SENSORY ACTION POTENTIAL RECORDED OVER THE RADIAL NERVE (Fig. 4) The electrodes placed over the radial nerve at the wrist recorded a potential of $2.5 \mu \mathrm{V}$ when supramaximal stimuli were applied to the distal phalanx of digit III. This is $12 \%$ of the potential recorded near the median nerve $(20 \pm 1 \cdot 7 \mu \mathrm{V}, \mathrm{N}=23)$. Activation of sensory nerve fibres of the radial nerve at the proximal part of the dorsum of digit III due to spread of the stimulating current is a possible cause of the potential recorded over the radial nerve, but in two patients with damage to the median nerve a potential over the radial nerve was not observed when stimulating the second phalanx of digit III.

The contamination of the radial potential by the potential from the median nerve was greater when the thumb than when digit III was stimulated. Considering that stimulation of the thumb evoked a potential of $40 \mu \mathrm{V}$ over the median nerve the pick-up by the radial electrodes amounted to abouf $5 \mu \mathrm{V}$; half of the spread is cancelled by the mode rejection of the input circuit (Buchthal and Rosen falck, 1966).

At the level of the elbow the distance between the radial and median nerves is about the same as at the wrist, but the potential picked up by the radia? electrodes was within the noise level unless electronic averaging was used. In fact, when stimulating digit III a potential was recorded over the radial nerve at the elbow in only one of 11 subjects. At the axilla the radial electrodes picked up a potential in two of seven subjects due to the shorter distance

TABLE I

CONDUCTION IN MOTOR FIBRES OF THE RADIAL NERVE AND AMPLITUDE OF MUSCLE ACTION POTENTIALS (35 $\left.{ }^{\circ} \mathrm{C}\right)$

\begin{tabular}{|c|c|c|c|c|c|c|c|c|}
\hline \multirow[t]{2}{*}{ Muscle } & \multirow[t]{2}{*}{ Conduction between } & \multirow[t]{2}{*}{ No. } & \multicolumn{2}{|c|}{$\begin{array}{l}\text { Conduction velocity } \\
(\mathrm{m} / \mathrm{sec}) \text { or conduction } \\
\text { time }(\mathrm{msec}) \ddagger\end{array}$} & \multicolumn{2}{|c|}{ Amplitude $(\mathrm{mV})$} & \multicolumn{2}{|c|}{ Distance $(\mathrm{cm})$} \\
\hline & & & Mean & $S . D$. & Mean & $S . D$. & Mean & S.D. \\
\hline \multirow{3}{*}{$\begin{array}{l}\text { Triceps brachii } \\
\text { Brachloradialis }\end{array}$} & axilla-muscle & 9 & $2 \cdot 7$ & 0.5 & 23 & $8 \cdot 1$ & $11 \cdot 3$ & 1.6 \\
\hline & axilla-elbow & 9 & 70 & $4 \cdot 9$ & 14 & $5 \cdot 7 *$ & $16 \cdot 2$ & $2 \cdot 7$ \\
\hline & elbow-muscle & 9 & $2 \cdot 5$ & $0 \cdot 3$ & 17 & $6 \cdot 1$ & $9 \cdot 5$ & $1 \cdot 1$ \\
\hline \multirow{2}{*}{ Extensor digitorum communis } & axilla-elbow & 11 & 69 & $5 \cdot 0$ & 13 & $4 \cdot 7^{*}$ & $18 \cdot 2$ & $2 \cdot 3$ \\
\hline & elbow-muscle & 17 & $2 \cdot 9$ & $0 \cdot 3$ & 16 & $6 \cdot 5$ & $9 \cdot 9$ & $1 \cdot 3$ \\
\hline \multirow[t]{3}{*}{ Extensor pollicis longus } & axilla-elbow & 6 & 67 & $8 \cdot 7$ & 15 & $5 \cdot 4^{*}$ & $16 \cdot 3$ & $2 \cdot 5$ \\
\hline & elbow-muscle & 14 & $4 \cdot 4$ & 0.6 & 14 & $6 \cdot 1$ & $19 \cdot 0$ & $2 \cdot 0$ \\
\hline & axilla-elbow & 8 & 69 & $5 \cdot 6$ & 11 & $7 \cdot 0^{*}$ & $15 \cdot 7$ & $3 \cdot 3$ \\
\hline \multirow{2}{*}{ Extensor indicis } & elbow-forearm & 10 & 62 & $5 \cdot 1$ & 13 & $8 \cdot 2 \dagger$ & $18 \cdot 1$ & $1 \cdot 5$ \\
\hline & forearm-muscle & 10 & $2 \cdot 4$ & 0.5 & 14 & $8 \cdot 8$ & $6 \cdot 2$ & 0.9 \\
\hline
\end{tabular}

*Stimulating at axilla.

+Stimulating at elbow.

$\ddagger$ Nerve-nerve recorded as conduction velocity. Nerve-muscle recorded as conduction time. 
between the median and radial nerves. Electronic averaging in three subjects showed that $25 \%$ of the potential at wrist and elbow and $50 \%$ of the potential at axilla originated from the median nerve (Fig. 4). Therefore the potential at the axilla is only slightly below that recorded at the elbow. When the radial nerve was stimulated at the wrist with needle or

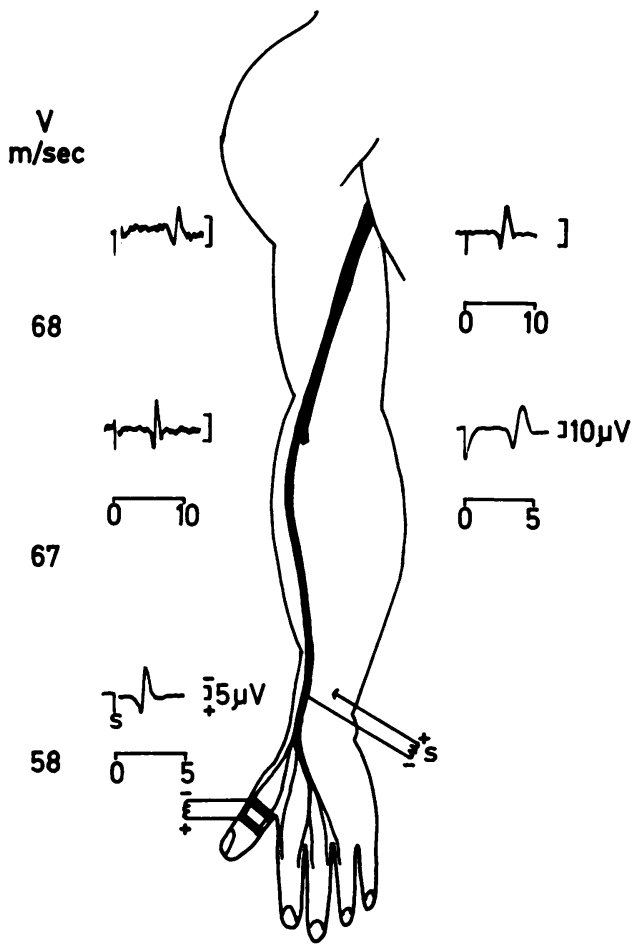

FIG. 3. Sensory action potentials recorded along the radial nerve at wrist, elbow and axilla by stimulating the thumb (left) and recorded at elbow and axilla by stimulating at wrist (right). Subject: J.P., female, 19 years old. Temperature near the nerve $35^{\circ} \mathrm{C}$. Stimulus $(S): 50 \mathrm{~mA}$ (left), $6 \mathrm{~mA}$ (right). Time scale in msec. surface electrodes, spread of the stimulus to the median nerve occurred when the stimulus exceeded 15 to $20 \mathrm{~mA}$ (Fig. 5).

\section{DISCUSSION}

The rate of motor conduction in fibres of the radial nerve between axilla and elbow was similar to the velocities in the nerve between the supraclavicular fossa and elbow (Gassel and Diamantopoulos, 1964; Tönnis, 1965). The conduction velocity to the extensor indicis muscle was $10 \%$ higher between axilla

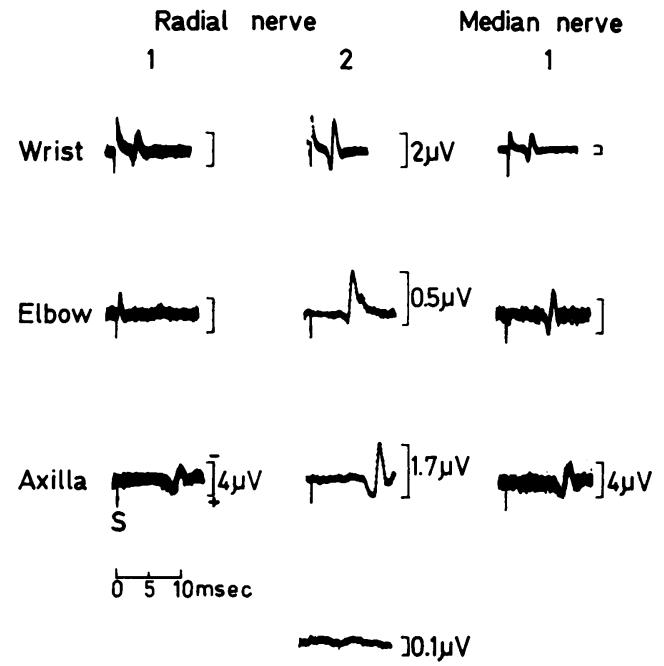

FIG. 4. Sensory action potentials from median nerve fibres recorded at the radial and median nerves at wrist, elbow and axilla evoked by stimulating the distal phalanx of digit III. (1) Photographic superposition of 12 to 15 sweeps, (2) electronic averaging of 700 responses. The lowermost trace is an average of 700 sweeps when leadingoff with stimulus zero. Subject: S.L.H., male, 17 years old. Temperature near the nerve $34^{\circ} \mathrm{C}$. Stimulus $(S): 40 \mathrm{~mA}$ $(12 \times t s$.$) .$

TABLE II

SENSORY CONDUCTION VELOCITY AND AMPLITUDE OF SENSORY ACTION POTENTIALS ALONG THE RADIAL NERVE $\left(35^{\circ} \mathrm{C}\right)$

\begin{tabular}{|c|c|c|c|c|c|c|c|c|c|c|c|c|c|c|c|c|c|c|}
\hline \multirow{4}{*}{ Stimulus to } & \multicolumn{9}{|c|}{ Conduction velocity $(\mathrm{m} / \mathrm{sec})$} & \multicolumn{9}{|c|}{ Amplitude $(\mu V)$} \\
\hline & & \multicolumn{3}{|c|}{ Thumb-wrist (1) } & \multirow{2}{*}{\multicolumn{2}{|c|}{ Wrist-elbow ${ }^{2}$}} & \multirow{3}{*}{ No. } & \multirow{2}{*}{\multicolumn{2}{|c|}{ Elbow-axilla $t^{2}$}} & \multirow{3}{*}{ No. } & \multirow{2}{*}{\multicolumn{2}{|c|}{ Wrist }} & \multirow{3}{*}{ No. } & \multirow{2}{*}{\multicolumn{2}{|c|}{ Elbow }} & \multirow{3}{*}{ No. } & \multirow{2}{*}{\multicolumn{2}{|c|}{ Axilla }} \\
\hline & \multirow[t]{2}{*}{ No. } & & & No. & & & & & & & & & & & & & & \\
\hline & & Mean & S.D. & & Mean & S.D. & & Mean & S.D. & & Mean & S.D. & & Mean & S.D. & & Mean & S.D. \\
\hline \multirow{3}{*}{$\begin{array}{l}\text { Thumb } \\
\text { Wrist } \\
\text { (needle electrodes) } \\
\text { Wrist } \\
\text { (surface electrodes) }\end{array}$} & 23 & $58^{*}$ & $6 \cdot 0$ & 20 & 69 & $5 \cdot 7$ & 16 & 71 & $5 \cdot 2$ & 23 & 13 & $7 \cdot 5$ & 20 & 5 & $2 \cdot 6$ & 16 & 4 & $1 \cdot 4$ \\
\hline & - & - & - & 13 & $66^{*}$ & $3 \cdot 5$ & 8 & 67 & $6 \cdot 5$ & - & - & - & 13 & 32 & $12 \cdot 1$ & 8 & 9 & $3 \cdot 0$ \\
\hline & - & - & - & 19 & $64^{*}$ & $6 \cdot 0$ & 7 & 68 & $4 \cdot 5$ & - & - & - & 19 & 31 & $11 \cdot 7$ & 7 & 10 & 3.6 \\
\hline
\end{tabular}

Distance in $\mathrm{cm}:$ (1) $13 \cdot 8 \pm 0 \cdot 4$. (2) $20 \cdot 0 \pm 0 \cdot 5$. (3) $18 \cdot 0 \pm 0 \cdot 7$.

${ }^{*}$ Calculated from the conduction time between stimulating cathode and recording electrode. 


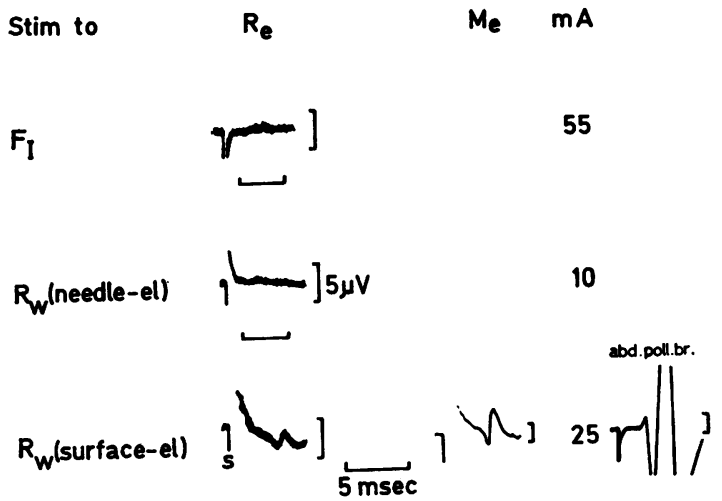

FIG. 5. 'False' response recorded over the radial nerve at elbow $(R e)$ in a patient with a lesion of the brachial plexus evoked by stimuli which are supramaximal in normal subjects $(17 \mathrm{~mA})$. The response, over the radial nerve was absent when stimulating the thumb $\left(F_{\mathrm{I}}\right)$ and when stimulating the nerve with needle electrodes at wrist $\left(R_{w}\right)$. When stimulating with surface electrodes at wrist a potential was recorded over the radial nerve. The simultaneously recorded potential over the median nerve $\left(M_{e}\right)$ was eight times greater, indicating spread of the stimulus to the median nerve at urist, as evidenced by the motor response in the abductor pollicis brevis muscle. Subject: I.P.N., male, 23 years old. Temperature near the nerve $35^{\circ} \mathrm{C}$.

and elbow than between elbow and forearm (Jebsen, 1966b). A similar difference between conduction rates in proximal and distal segments of the median, ulnar, and sciatic nerves has been described (Gassel and Trojaborg, 1964; Trojaborg, 1964).

The conduction velocity in sensory fibres of the radial nerve was the same in the proximal and intermediate segments of the nerve similar to findings in the median and ulnar nerves (Buchthal and Rosenfalck, 1966). The sensory conduction velocity between wrist and elbow was about $20 \%$ higher than found by Downie and Scott $(1964,1967)$, who used the peak of the negative spike as point of reference to calculate conduction velocity. The slope of the positive-negative deflection increases with increasing distance from the stimulating electrode.

The $60 \%$ decrease in amplitude from wrist to elbow when stimulating the thumb and the similar decrease from elbow to axilla when stimulating the nerve at the wrist correspond well to the decrease in amplitude on account of temporal dispersion reconstructed from the compound action potential (Buchthal and Rosenfalck, 1966).

Stimulating the radial nerve at the wrist instead of at the thumb had the double advantage that spread from the median nerve was avoided and that the potential at the elbow and the axilla was about six times greater because of the larger number of fibres at the wrist and because of the shorter distance of conduction.

When stimulating the thumb the contamination of the potential recorded above the radial neive by potentials spread from the median nerve is inevitable and makes it impossible to decide whether or not there is a complete block of the radial nerve distal to the wrist. Stimulation of the radial nerve at the wrist provides information about the intermediate and proximal portions of the nerve. However, even when the radial nerve is stimulated at the wrist, care must be taken that the stimulus does not spread to the median nerve, as may easily happen when the electrode is a 4 to $5 \mathrm{~cm}$ long 'pipe cleaner' laid around the radial aspect of the wrist (Downie and Scott, 1964). When needle electrodes are placed along the radial nerve, spread of the stimulus to the median nerve is minimal.

The organization of sensory fibres in the radial nerve might partly account for the variation in amplitude of action potentials recorded over the nerve at the elbow. The sensory fibres can be stripped nearly $7 \mathrm{~cm}$ above the division of the radial nerve into the posterior interosseous and the superficial branches without interfering with the fibre? architecture of the nerve, and in the main trunk at 0 the level of the intermuscular furrow sensory fibres are localized to the anteromedial and central part of the nerve (Sunderland, 1945). If this is correct, the amplitude of the sensory potential would depend on whether the recording electrode were placed? lateral or medial to the nerve. Hence the threshold of the antidromically conducted sensory potential might be a more reasonable guide for correct positioning of the recording electrode at the elbow.

\section{SUMMARY}

Motor and sensory nerve conduction in the radial nerve was determined in normal subjects 16 to 28 years old. The conduction velocities of motor and sensory fibres were the same in the proximal segment of the nerve (axilla to elbow, $69 \mathrm{~m} / \mathrm{sec}$ ), of the same order as in the median nerve. The motor velocity was $10 \%$ lower in the intermediate than in the proximal segments of the nerve whereas the sensory conduction velocity was the same. The sensory velocity between the thumb and wrist was $17 \%$ lower than the proximal velocities.

When stimulating the thumb $25 \%$ of the sensory potential over the radial nerve at wrist and elbow and $50 \%$ at the axilla was due to spread from median nerve fibres. When the stimulus was applied through needle electrodes to the radial nerve the contamination from the median nerve fibres could be 
avoided as long as the stimulating current was less than $15 \mathrm{~mA}$.

\section{REFERENCES}

Andersen, V. O. (1966). Annual Report of the Institute of Neurophysiology of the University of Copenhagen. Universitetsforlaget : Copenhagen.

Buchthal, F., and Rosenfalck, A. (1966). Evoked action potentials and conduction velocity in human sensory nerves. Brain Res., 3, 1-119.

Downie, A. W., and Scott, T. R. (1964). Radial nerve conduction studies. Neurology (Minneap.), 14, 839-843.

(1967). An improved technique for radial nerve conduction studies. J. Neurol. Neurosurg. Psychiat., 30, 332-336.
Gassel, M. M., and Diamantopoulos, E. (1964). Pattern of conduction times in the distribution of the radial nerve. A clinical and electrophysiological study. Neurology (Minneap.), 14, 222-231.

study of the pattern of conduction times in the distribution of the sciatic nerve. J. Neurol. Neurosurg. Psychiat., 27, 351-357.

Jebsen, R. H. (1966a). Motor conduction velocity of distal radial nerve. Arch. phys. Med., 47, 12-16.

- (1966b). Motor conduction velocity in proximal and distal segments of the radial nerve. Ibid., 47, 597-602.

Sunderland, S. (1945). Intraneural topography of the radial, median and ulnar nerves. Brain, 68, 243-299.

Trojaborg, W. (1964). Motor nerve conduction velocities in normal subjects with particular reference to the conduction in proximal and distal segments of median and ulnar nerves. Electroenceph. clin. Neurophysiol., 17, 314-321.

Tönnis, D. (1965). Untersuchungen über die Leitgeschwindigkeit des N. radialis. Z. Orthop., 99, 497-507. 